\title{
Analytical Design Equations for Class-E Power Amplifiers with Finite DC-Feed Inductance and Switch On-Resistance
}

\author{
Mustafa Acar, Anne Johan Annema and Bram Nauta \\ IC Design Chair, University of Twente, The Netherlands
}

\begin{abstract}
Many critical design trade-offs of the Class-E power amplifier (e.g power efficiency) are influenced by the switch onresistance and the value of dc-feed drain inductance. In literature, the time-domain mathematical analyses of the Class- $E$ power amplifier with finite dc-feed inductance assume zero switch onresistance in order to alleviate the mathematical difficulties; resulting in non-optimum designs.

We present analytical design equations in this paper for Class-E power amplifier taking into account both finite drain inductance and switch on-resistance. The analysis indicates the existence of infinitely many design equations; conclusions include:

1) Class-E conditions (e.g. zero voltage and zero slope) can be satisfied in the presence of switch-on resistance.

2) The drain-efficiency $(\eta)$ of the Class-E power amplifier is upper limited for a certain operation frequency and transistor technology.
\end{abstract}

3) Using a finite dc-feed inductance instead of an RF-choke in a Class-E power amplifier can increase $\eta$ by $\approx 30 \%$.

\section{INTRODUCTION}

The Class-E power amplifier (PA) has been very popular due to it's high efficiency and the simple circuit structure [1]. However, the "finite dc-feed inductance" and the "non-zero switch on-resistance" significantly influence the performance of the Class-E PAs [2]. To alleviate the analytical complexity, theoretical analyses of the Class-E PA in the literature assumed either non-zero switch-on resistance and infinite dc-feed inductance (RF-choke) [3]- [7] or zero switch-on resistance and finite dc-feed inductance [8]- [11].

It is well-known that using a finite dc feed inductance instead of an RF-choke in Class-E has benefits [8], [9] including:

- a reduction in overall size and cost

- a higher load resistance for the same supply voltage and output power; yielding more efficient output matching networks

- larger switch parallel capacitor $C$ (Fig.1a) for the same supply voltage, output power and load; enabling higher drain efficiency or higher frequency of operation.

In order to design Class-E PAs with optimum performance an improved analytical model that takes into account both the finite drain inductance and non-zero switch on-resistance is therefore needed [2].

In [12]- [21] the switch on-resistance is taken into account in the design of Class-E PAs. In [12], the shunt capacitor $(C$ see Fig. 1b) is assumed to be disconnected at the switch turn-on moment; which makes the analysis only an approximation. In [13], the shunt capacitor voltage $\left(V_{C}(t)\right)$ is assumed to be zero at the switch turn-off moment; which is not analytically exact and can be accepted only for very small switch on-resistance $\left(R_{\text {on }}<<R\right)$.

The analysis and the design approach given in [14] offers no initial design guidelines, which tends to make it tedious because of the inherently large number of iterations that are required [5].

Moreover, the design methodologies presented in [15]- [20] either relies on iteration [15]- [19] or assigning initial values to some design variables [20]. In [21], an analytical solution for a sub-class of Class-E PA ${ }^{1}$ is presented.
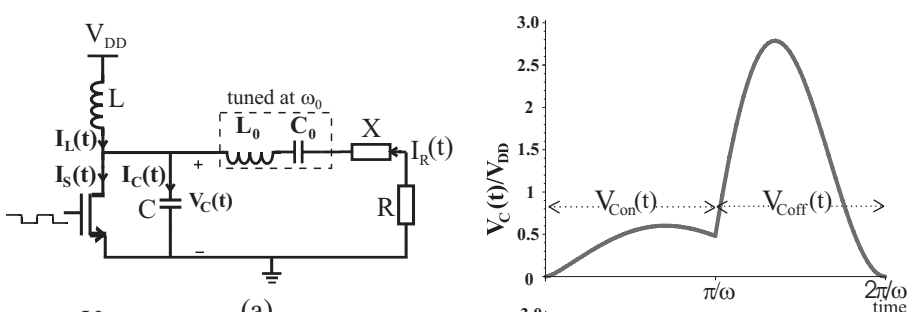

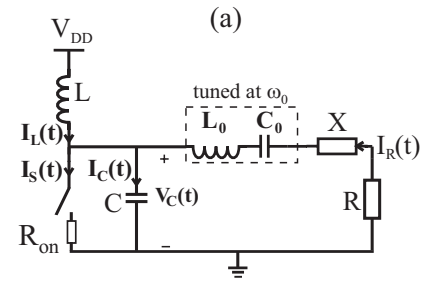

(b)

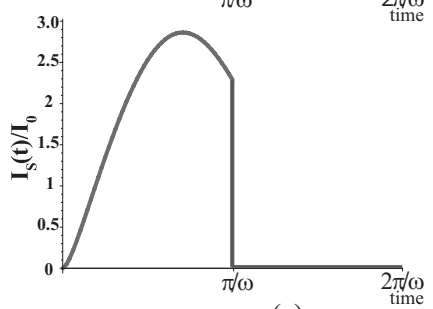

(c)
Fig. 1. (a) Single-ended Class-E PA (b) Model of Class-E PA with finite dcfeed inductance and switch on-resistance (c) Normalized switch (transistor) voltage and current for the model of Class-E PA

This paper presents an analysis (time domain) and design equations for Class-E PAs with finite dc-feed inductance and non-zero switch on-resistance. The analysis in this paper is based on closed form expressions like those presented in [9] and $[10]^{2}$. The analysis yields analytical design equations that show the relation between the various design parameters.

${ }^{1}$ The Class-E topology given in [21] assumes zero switch parallel capacitor; which is only applicable for very dedicated technologies with very small $R_{\text {on }} C_{\text {out }}$ product, where $C_{\text {out }}$ is the switch (transistor) output capacitance.

${ }^{2}$ In [9] and [10], the switch on-resistance is assumed to be zero. 


\section{ANALYSis OF Class-E POWER AMPlifieR}

A single ended switching PA topology is given in Fig.1a. For correct input parameters and the circuit element values, the circuit properly operates as a Class-E PA by satisfying the following conditions (1) [9]:

$$
V_{C}(2 \pi / \omega)=0 \quad \text { and }\left.\quad \frac{d V_{C}(t)}{d t}\right|_{t=2 \pi / \omega}=0
$$

A design set $K=\left\{K_{L}, K_{C}, K_{P}, K_{X}\right\}$ (see Table-1) that relates circuit element values to operating conditions such as supply voltage, operating frequency and output power for the switching PA in Fig.1b can be derived. In [9], an analytical solution for $K$ is given that enables infinitely many ideal Class-E realizations, to be selected by one parameter $q=$ $\frac{1}{\omega \sqrt{L C}}$. In this paper, one more step is taken and the switch on-resistance $R_{o n}$ is included in the analysis. As it is shown (later) in this section the design set $K$ can be expressed as a function of only two parameters $q$ and $m=\omega R_{o n} C$ both of which are free design variables and can take any positive real value.

$$
K_{L}=\frac{\omega L}{R}, K_{C}=\omega C R, K_{P}=\frac{P_{O U T} R}{V_{D D}^{2}}, K_{X}=\frac{X}{R}
$$

Table 1: Design Set $K$ for Class-E PA ${ }^{3}$

As mentioned, the analytical solution in [9] is extended to cover Class-E PAs including $R_{O N}$ in this paper.

\section{A. Circuit Description and Assumptions}

The circuit model of the Class-E PA is given in Fig.1b. For the analysis and the derivations in this paper a number of assumptions are made:

- the only real power loss occurs on $R$ and $R_{\text {on }}$

- the switch (transistor) operates instantly with onresistance $\left(R_{o n}\right)$ and infinite off-resistance

- the loaded quality factor $\left(Q_{L}\right)$ of the series resonant circuit $\left(L_{0}\right.$ and $\left.C_{0}\right)$ is high enough in order for the output current to be sinusoidal at the switching frequency

- the duty cycle is $50 \%$

Fig.1c shows the switching behavior and the switch definition used: in the time interval $0 \leq t<\pi / \omega$ the switch is closed and in $\pi / \omega \leq t<2 \pi / \omega$ it is opened. This switching repeats itself with a period of $2 \pi / \omega$.

\section{B. Circuit Analysis}

In the analysis, the current into the load, $I_{R}(t)$, is assumed to be sinusoidal. Note that theoretically this occurs only for infinite $Q_{L}$ of the series resonant network consisting of $L_{0}$ and $C_{0}$. It is however a widely used assumption [8], [9], [12] that simplifies analysis considerably:

$$
I_{R}(t)=I_{R} \sin (\omega t+\varphi)
$$

In the time interval $0<t<\pi / \omega$, the switch is closed. The $\mathrm{KCL}$ at the drain node can be written as:

$$
I_{L}(t)-I_{S}(t)-I_{C}(t)+I_{R}(t)=0
$$

${ }^{3} L_{0}$ and $C_{0}$ seen in Fig. 1 can be determined from the chosen loaded quality factor $\left(Q_{L}=\omega_{0} L_{0} / R\right)$ where $\omega_{0}=1 / \sqrt{L_{0} C_{0}}$.
Relation (3) can be arranged in the form of a linear, nonhomogenous, second order differential equation

$$
\begin{aligned}
C \frac{d^{2} V_{C_{o n}}(t)}{d t^{2}}+\frac{1}{R_{o n}} & \frac{d V_{C_{o n}}}{d t}-\frac{V_{D D}-V_{C_{o n}}}{L} \\
& -\omega I_{R} \cos (\omega t+\varphi)=0
\end{aligned}
$$

which has as solution

$$
\begin{aligned}
V_{C_{o n}}= & \frac{\left(q^{4} \sin (\omega t+\varphi) m+\left(-q^{2}+q^{4}\right) \cos (\omega t+\varphi)\right) p V_{D D}}{1+\left(m^{2}+1\right) q^{4}-2 q^{2}} \\
& +V_{D D}+e^{a \omega t} C_{o n_{2}}+e^{b \omega t} C_{o n_{1}}
\end{aligned}
$$

where, $a=\frac{-1+\sqrt{1-4 q^{2} m^{2}}}{2 m}, b=\frac{-1-\sqrt{1-4 q^{2} m^{2}}}{2 m}$ and $p=$ $\frac{\omega L I_{R}}{V_{D D}} . C_{o n_{1}}$ and $C_{o n_{2}}$ follow from the continuity of the capacitor voltage $(C)$ and the inductor $(L)$ current at the switch-on moment.

In the time interval $\pi / \omega<t<2 \pi / \omega$, the switch is opened. Then, in the Class-E PA the current through capacitance $C$ is

$I_{C}(t)=\frac{1}{L} \int_{\pi / \omega}^{t}\left(V_{D D}-V_{C_{o f f}}(t)\right) d t+I_{L}\left(\frac{\pi}{\omega}\right)+I_{R}(t)$

Relation (6) can be re-arranged in the form of a linear, nonhomogeneous, second-order differential equation

$$
L C \frac{d^{2} V_{C_{o f f}}(t)}{d t^{2}}+V_{C_{o f f}}(t)-V_{D D}-\omega L I_{R} \cos (\omega t+\varphi)=0
$$

which has as solution

$$
\begin{aligned}
V_{C_{o f f}}(t)= & C_{o f f_{1}} \cos (q \omega t)+C_{o f f_{2}} \sin (q \omega t)+V_{D D} \\
& -\frac{q^{2}}{1-q^{2}} p V_{D D} \cos (\omega t+\varphi)
\end{aligned}
$$

$C_{o f f_{1}}$ and $C_{o f f_{2}}$ follow from the Class-E conditions (1).

It follows from (5) and (8) that $V_{C_{o n}}(t)$ and $V_{C_{o f f}}(t)$ can be expressed in terms of $V_{D D}$ and $\omega$ hence be solved analytically only if $\varphi, q, p$ and $m$ are known. The derivation of the four parameters $\varphi, p, q$ and $m$ is the next step in the solution.

By using the continuity of the inductor current and the capacitor voltage at the switch turn-off moment we can derive two independent equations which can be shown to have the same format:

$$
f_{i}(p, q, \varphi, m)=p\left(a_{i}(q, m) \cos (\varphi)+b_{i}(q, m) \sin (\varphi)\right)+
$$
$c_{i}(q, m)=0$, where $i=1,2$.

The variables $p$ and $\varphi$ can be solved by using $f_{1}(p, q, \varphi, m)$ and $f_{2}(p, q, \varphi, m)$ in terms of $q$ and $m$ as given in the appendix. Here, $q$ and $m$ are free variables that can mathematically take any positive real value.

\section{Design sets for Class-E operation}

The results of the mathematical derivation of the solutions leading to Class-E operation can be used to derive an an easy-to-use design procedure for Class-E PAs. Using the result of the derivation for $p(q, m)$ and $\varphi(q, m)$, analytical expressions for the design set $K=\left\{K_{L}, K_{C}, K_{P}, K_{X}\right\}$ can readily be derived. 


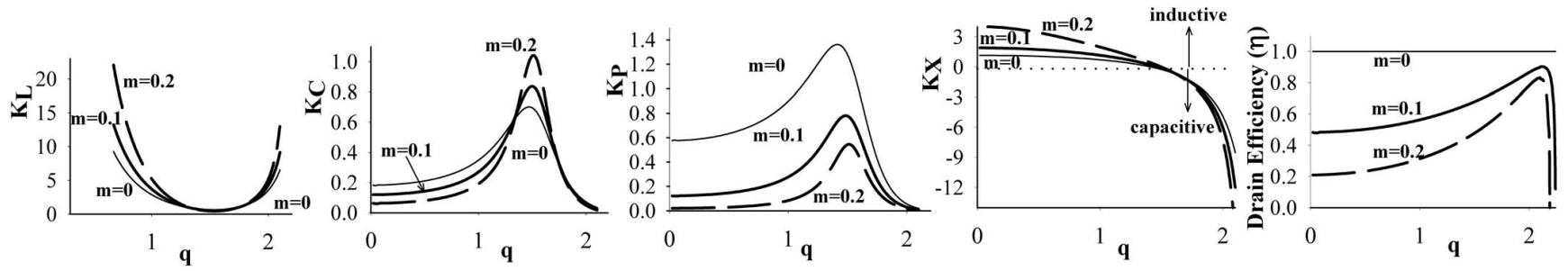

Fig. 2. (a) Design set $K$ and drain efficiency $(\eta)$ as a function of $q$ for $m=0,0.1,0.2$.

$K_{L}$ : follows from the principle of power conservation:

$$
I_{R}^{2} R / 2+P_{\text {switch }}=I_{0} V_{D D}
$$

In this relation, $I_{0}$ is the average supply current:

$$
I_{0}=\frac{\omega}{2 \pi R_{o n}} \int_{0}^{\pi / \omega} V_{C_{o n}}(t) d t
$$

and,

$$
P_{\text {switch }}=\frac{\omega}{2 \pi R_{o n}} \int_{0}^{\frac{\pi}{w}}\left(V_{C_{o n}}(t)\right)^{2} d t
$$

Substitution of (10) and $p$ in (9) yields

$$
K_{L}(q, m)=\frac{-\left(p V_{D D} q\right)^{2} m \pi}{\omega \int_{0}^{\frac{\pi}{w}}\left(V_{C_{o n}}(t)^{2}-V_{D D} V_{C_{o n}}(t)\right) d t}
$$

Since $p$ and $\varphi$ are all functions of $q$ and $m, K_{L}(q)$ is a function of e.g. only $q$ and $m$.

$K_{C}$ : follows directly from the definition of $q$ and $K_{L}$ :

$$
K_{C}(q, m)=\frac{1}{q^{2} K_{L}(q, m)}
$$

$K_{P}$ : can easily be found as a function of $q$ and $m$ by using $I_{R}=\sqrt{2 P_{\text {OUT }} / R}$ and the definition of $p$ :

$$
K_{P}(q, m)=p(q, m)^{2} /\left(2 K_{L}(q, m)^{2}\right)
$$

$K_{X}$ : can be derived using two fundamental quadrature Fourier components of $V_{C}(t)$.

$V_{R}=\int_{0}^{\frac{\pi}{\omega}} \frac{V_{C_{o n}}(t)}{\pi} \sin (\omega t+\varphi) d t+\int_{\frac{\pi}{\omega}}^{\frac{2 \pi}{\omega}} \frac{V_{C_{o f f}}(t)}{\pi} \sin (\omega t+\varphi) d t$

$V_{X}=\int_{0}^{\frac{\pi}{\omega}} \frac{V_{C_{o n}}(t)}{\pi} \cos (\omega t+\varphi) d t+\int_{\frac{\pi}{\omega}}^{\frac{2 \pi}{\omega}} \frac{V_{C_{o f f}}(t)}{\pi} \cos (\omega t+\varphi) d t$

$$
K_{X}(q, m)=V_{X} / V_{R}
$$

Drain efficiency $(\eta)$ : can be derived as a function of $q$ and $m$.

$$
\eta(q, m)=1-\frac{P_{\text {switch }}}{V_{D D} I_{0}}=1-\frac{\int_{0}^{\frac{\pi}{w}}\left(V_{C_{o n}}(t)^{2}\right) d t}{V_{D D} \int_{0}^{\frac{\pi}{\omega}} V_{C_{o n}}(t) d t}
$$

We verified the given design equations in this paper by simulating the model given in Fig.1b by transient and pss

\begin{tabular}{c||c|c|c}
\hline Design Details & RF-choke $(\mathbf{q}=\mathbf{0})$ & finite $(\mathbf{q}=\mathbf{1 . 4 7})$ & finite $(\mathbf{q = 1 . 7 8})$ \\
\hline \hline $\mathrm{f}(\mathrm{GHz}), V_{D D}(\mathrm{~V}), Q_{L}$ & $2.4,0.5,10$ & $2.4,0.5,10$ & $2.4,0.5,10$ \\
\hline \hline$P_{O U T} / P_{D C}(\mathrm{~mW})$ & $10.6 / 22.2$ & $11.8 / 17.0$ & $12.1 / 15.2$ \\
m, Drain Efficiency $(\eta)$ & $\mathbf{0 . 1}, \mathbf{4 7 . 7 \%}$ & $\mathbf{0 . 1}, \mathbf{6 9 . 4 \%}$ & $\mathbf{0 . 1}, \mathbf{7 9 . 6 \%}$ \\
$\mathbf{L}, L_{X}(\mathrm{nH}), C(\mathrm{pF})$ & $\mathbf{2 0 . 2 9}, 0.38,2.59$ & $\mathbf{0 . 7 2}, 0,2.82$ & $\mathbf{0 . 4}-, 3.48$ \\
$\mathbf{R}(\Omega), C_{X}(\mathrm{nF})$ & $\mathbf{3 . 0 6},-$ & $\mathbf{1 9 . 4 7},-$ & $\mathbf{5 . 0 6}, 6.88$ \\
$(\mathrm{~W}(\mathrm{u}) / \mathrm{L}(\mathrm{u})), K_{L}$ & $(297 / 0.1), 100$ & $(323 / 0.1), 0.56$ & $(398 / 0.1), 1.19$ \\
$K_{C}, K_{P}, K_{X}$ & $0.12,0.12,1.89$ & $0.83,0.78,0$ & $0.27,0.20,-1.90$ \\
Technology & $90 \mathrm{~nm}$ CMOS & 90nm CMOS & $90 \mathrm{~nm} \mathrm{CMOS}$ \\
\hline \hline
\end{tabular}

Table 2: Comparison and design summary of the three Class-E PA designs for $m=0.1$ and $q=0,1.47,1.78$ in CMOS 90nm transistor technology

(periodic steady state) simulations in spectre (cadence). Very good agreement in the waveforms and the drain efficiency are observed between the simulations and the theory with a discrepancy of $\approx 2 \%$; attributed to the finite value of $Q_{L}=10$.

In theory, $q$ can take any positive real number however, as it is seen in Fig. $2 K_{C}, K_{P}$ and $\eta$ approach to zero for $q>2$. Therefore, the useful range of the analytical solution can be assumed to be restricted to $0<q<2$ in Class-E PA designs. Similarly, as $m$ increases $K_{P}$ and $\eta$ drops as observed in Fig.2; indicating the degradation in Class-E PA performance.

\section{DESIGN EXAMPLES AND DISCUSSION}

The analytical design equations reveal very important properties of the Class-E PAs. For example, we can express $m \approx$ $\beta \omega$ where $\beta=R_{\text {on }} C_{\text {out }}$. $\beta$ is a characteristic property of the transistor technology used as a switch in Class-E PA design ${ }^{4}$. For a certain operation frequency and transistor technology $m$ has a certain value. As it is seen in Fig.2, there is a maximum efficiency level that could be achieved for a given $m$; showing that the transistor technology and the frequency of operation $d t$ sets an upper limit for $\eta$ of a Class-E PA.

The chosen value of $q$ considerably influence $\eta$ as observed from Fig. 2 and the simulation results given in Table-2. We designed three Class-E PAs for an output power of $10 \mathrm{~mW}^{5}$. Finite dc-feed Class-E PA( $\mathrm{q}=1.78)$ has $\eta$ that is $\approx 30 \%$ higher than RF-coke Class-E $\operatorname{PA}(q=0)$; indicating how much $\eta$ can be influenced by the chosen design equations.

\footnotetext{
${ }^{4}$ In order to minimize $R_{o n}$, maximum possible transistor size can be chosen for which transistor output capacitance $C_{\text {out }}=C$.

${ }^{5}$ Slightly higher output power than $10 \mathrm{~mW}$ is attributed mostly to deviation of transistor characteristic from an ideal switch behavior at high frequency.
} 
Although the Class-E $\operatorname{PA}(q=1.47)$ has lower $\eta$ than the Class-E $\operatorname{PA}(q=1.78)$ it's load resistance $(R)$ is $\approx 4$ times higher than that of the Class-E $\operatorname{PA}(q=1.78)$; which is very advantageous for low supply voltage - high output power Class-E amplifiers ${ }^{6}$

The Class-E $\operatorname{PA}(q=1.78)$ can be used for low power applications (e.g. wireless sensors) where the transmit power levels are low $\approx(1-3) m W[22]$ and high efficiency is crucial. If the Class-E $\operatorname{PA}(q=1.78)$ is designed for an output power of $1 \mathrm{~mW}$, it needs $R \approx 50 \Omega$; meaning that a matching network between the PA and the antenna is not needed.

\section{CONCLUSION}

In this paper, we present a time domain analysis and closed form analytical design equations for Class-E power amplifiers with finite dc-feed inductance and non-zero switch on-resistance. Important outcomes of the analysis include:

1) Class-E conditions (e.g zero voltage and zero slope) can be satisfied in the presence of the switch on-resistance.

2) Drain efficiency $(\eta)$ for Class-E PAs is upper limited by the transistor technology and the operation frequency.

3) Using a finite dc-feed inductance instead of an RFchoke in Class-E PAs increases $\eta$. Depending on the transistor technology and the operation frequency the increase in $\eta$ can be as high as $\approx 30 \%$.

\section{REFERENCES}

[1] N. O. Sokal and A. D. Sokal, "Class E-A new class of high-efficiency tuned single-ended switching power amplifiers" IEEE JSSC, vol. SC-10, pp. 168-176, June 1975.

[2] Lie, D.Y.C. et al. "The limitations in applying analytic design equations for optimal class E RF power amplifiers design" VLSI Design, Automation and Test, IEEE 2005, pp:161 - 164

[3] D. K. Choi and S. I. Long, "A Physically Based Analytic Model of FET Class E Power Amplifiers Designing for Maximum PAE," IEEE Trans. on Microwave Theory and Techniques, vol. 47,pp. 1712-1720 1999

[4] Kessler, D.J.; Kazimierczuk, M.K. ”Power losses and efficiency of class-E power amplifier at any duty ratio"/emphCircuits and Systems I: Regular Papers, IEEE Transactions on [Circuits and Systems I: Fundamental Theory and Applications, IEEE Transactions on] vol: 51, pp: 1675-1689, Sept. 2004

[5] D. K. Choi "High Eficiency Switched-Mode Power Amplifiers For Wireless Communications" PhD thesis, page 121.

[6] Alinikula, P.; "Optimum component values for a lossy Class E power amplifier" International Microwave Symposium Digest, IEEE vol.3, June 2003 pp:2145 - 2148

[7] Raab, F.H.; Sokal, N.O.; "Transistor power losses in the class E tuned power amplifier" IEEE JSSC vol. 13, Dec 1978 pp:912 - 914

[8] R. Zulinski, J. Steadman, "Class E Power Amplifiers and Frequency Multipliers with finite DC-Feed Inductance," IEEE Trans. on CAS, vol. 34, Sep 1987, pp. 1074-1087

[9] M. Acar, A.J. Annema, B. Nauta "Generalized Design Equations for Class-E Power Amplifiers with Finite DC Feed Inductance" 36th European Microwave Conference, September 2006, pp. 13081311.

[10] M. Acar, A.J. Annema, B. Nauta "Generalized Analytical Design Equations for Variable Slope Class-E Power Amplifiers" 13th IEEE International Conference on Electronics, Circuits and Systems, December 2006, accepted for publication.

[11] Choi, D.K.; Long, S.I.; "Finite DC feed inductor in class E power amplifiers-a simplified approach" International Microwave Symposium Digest, IEEE vol. 3, June 2002 pp:1643-1646

${ }^{6}$ In order to obtain high output power from low supply voltage Class-E PAs a matching network that steps down $50 \Omega$ antenna impedance to low impedance values is used. In the absence of high $\mathrm{Q}$ inductors the matching network can be very lossy for high transformation ratios.
[12] Wang, C.; Larson, L.E.; Asbeck, P.M.; "Improved design technique of a microwave class-E power amplifier with finite switching-on resistance" Radio and Wireless Conference, IEEE, Aug. 2002, pp:241-244

[13] Ho, C.K.; Wong, H.; Ma, S.W.; "Approximation of non-zero transistor ON resistance in class-E amplifiers" Proceedings of the Fifth IEEE International Caracas Conference on, vol. 1, Nov. 2004, pp:90-93

[14] Avratoglou, C.P.; Voulgaris, N.C.; Ioannidou, F.I.; "Analysis and design of a generalized class E tuned power amplifier" Circuits and Systems, IEEE Transactions on vol. 36, Aug. 1989, pp:1068-1079

[15] Sekiya, H.; Sasase, I.; Mori, S.; "Computation of design values for Class E amplifiers without using waveform equations" Circuits and Systems I: Fundamental Theory and Applications, IEEE Transactions on vol. 49, July, 2002, pp:966-978

[16] Reynaert, P.; Mertens, K.L.R.; Steyaert, M.S.J.; "A state-space behavioral model for CMOS class E power amplifiers" Computer-Aided Design of Integrated Circuits and Systems, IEEE Transactions on, vol. 22, Feb. 2003, pp:132-138

[17] Mandojana, J.C.; Herman, K.J.; Zulinski, R.E.; ”A discrete/continuous time-domain analysis of a generalized class E amplifier" Circuits and Systems, IEEE Transactions on vol. 37, Aug. 1990, pp:1057 - 1060

[18] Choi, Y.-B.; Cheng, K.-K.M.; "Generalised frequency-domain analysis of microwave Class-E power amplifiers" Microwaves, Antennas and Propagation, IEE Proceedings, vol. 148, Dec. 2001, pp:403-409

[19] Tabrizi, M.M.; Masoumi, N.; "High efficiency class-E switched mode power amplifier design and optimization with random search algorithm" Microelectronics ICM Proceedings, 2004, pp:283 - 286

[20] J.-K. Jau, Y.-A. Chen, T.-S. Horng, and T.-L. Wu "Optimum Analytical Design Solution to Integrated Class-E Amplifiers" Proceeding of Wireless Networks and Emerging Technologies - 2005

[21] Mury, T.; Fusco, V.F.; "Analysis and synthesis of pHEMT class-E amplifiers with shunt inductor including ON-state active-device resistance effects" Circuits and Systems I: Regular Papers, IEEE Transactions on, vol. 53, July 2006, pp:1556-1564

[22] Chee, Y.H.; Rabaey, J.; Niknejad, A.M.; "A class A/B low power amplifier for wireless sensor networks" Proceedings of International Symposium on Circuits and Systems, vol. 4, May 2004 pp: 409-412

\section{APPENDIX I}

In this section the solution for $p$ and $\varphi$ in terms of $q$ and $m$ are given ${ }^{7}$.

$$
\begin{aligned}
p= & \frac{\sqrt{\left(g_{1} h_{3}-g_{3} h_{1}\right)^{2}+\left(h_{2} g_{3}-h_{3} g_{2}\right)^{2}}}{-g_{1} h_{2}+g_{2} h_{1}}, \varphi=\arctan \left(h_{2} g_{3}-h_{3} g_{2}, g_{1} h_{3}-g_{3} h_{1}\right) \\
g_{1}= & \frac{-e^{a \pi}\left(A+b m q^{2}\right)+e^{b \pi}\left(A+a m q^{2}\right)}{B(-b+a)}-\frac{q \sin (q \pi)}{q^{2}-1}+\frac{m q^{2}}{B} \\
g_{2}= & -\frac{(\cos (q \pi)+1) q^{2}}{q^{2}-1}+\frac{m^{2} q^{2}(q-1)(q+1)}{B}+ \\
& \frac{-e^{a \pi}\left(A b-m q^{2}\right)+e^{b \pi}\left(A a-m q^{2}\right)}{B(-b+a)} \\
g_{3}= & \frac{e^{b \pi} a-e^{a \pi} b}{-b+a}-\cos (q \pi) \\
h_{1}= & \frac{m^{3} q^{2}(q-1)(q+1)}{B}-\frac{q(m \cos (q \pi) q+\sin (q \pi)+m q)}{q^{2}-1} \\
& +m\left(\frac{a e^{a \pi}\left(A+b m q^{2}\right)}{B(-b+a)}-\frac{b e^{b \pi}\left(A+m q^{2} a\right)}{B(-b+a)}\right) \\
h_{2}= & -\frac{m^{2} q^{2}}{B}+\frac{q^{2}(m \sin (q \pi) q-\cos (q \pi)-1)}{q^{2}-1} \\
& +m\left(\frac{a e^{a \pi}\left(A b-m q^{2}\right)}{B(-b+a)}-\frac{b e^{b \pi}\left(A a-m q^{2}\right)}{B(-b+a)}\right) \\
h_{3}= & -\cos (q \pi)+m \sin (q \pi) q+m\left(\frac{a e^{a \pi} b}{-b+a}-\frac{b e^{b \pi} a}{-b+a}\right)+1 \\
A= & \left(q^{4}-q^{2}\right) m^{2} \text { and } B=1+m^{2} q^{4}-2 m^{2} q^{2}+m^{2}
\end{aligned}
$$

\footnotetext{
${ }^{7}$ Two roots exist for $p$ and $\varphi$. The second root is $p^{\prime}=-\mathrm{p}$ and $\varphi^{\prime}=$ $\arctan \left(-h_{2} g_{3}+h_{3} g_{2},-g_{1} h_{3}+g_{3} h_{1}\right)$. Both roots result in the same K.
} 